\title{
Dependence of pseudomorphic semiconductor band gap on substrate orientation
}

\author{
J. M. Hinckley and J. Singh \\ Center for High Frequency Microelectronics, Department of Electrical Engineering and Computer Science, \\ The University of Michigan, Ann Arbor, Michigan 48109-2122
}

(Received 20 August 1990; accepted for publication 29 October 1990)

\begin{abstract}
For a given misfit we examine the band-gap variation of a pseudomorphic overlayer on a thick substrate as a function of substrate orientation. The strain tensor is found to be a strong function of the substrate orientation. For both direct and indirect band-gap overlayers, this results in a significant variation in the band gap as the substrate orientation is changed. However, for indirect band-gap layers, such as $\mathrm{SiGe}$ alloys grown on $\mathrm{Si}$ substrates, the change in band gap is accompanied by a lifting of conduction-band-edge degeneracies. The magnitude of this splitting may be as large or larger than the change in the band gap.
\end{abstract}

The ability to incorporate a fairly large strain in active regions of semiconductor devices by pseudomorphic growth has introduced an important tool in band-gap engineering. Not only does the built-in strain change the band gap of the epitaxial film significantly, but the accompanying reduction of symmetry can also lift band-edge degeneracies, leading to important changes in optical and electronic properties. To realize these benefits, strained epitaxy is being pursued in both direct band-gap materials (e.g., $\mathrm{In}_{x} \mathrm{Ga}_{1-x}$ As on $\mathrm{GaAs}$ or InP substrates) as well as in indirect materials (e.g., $\mathrm{Si}_{1}{ }_{x} \mathrm{Ge}_{x}$ on $\mathrm{Si}$ substrates). Much of the current work focuses on the (001) substrate orientation because of its general compatability with both growth and fabrication technology. Nevertheless, it must be realized that the pseudomorphic strain tensor changes significantly as the substrate orientation changes. As a result, one can expected corresponding changes in the band gap and other electronic properties. It may be pointed out that by proper substrate cutting and patterning, it is possible to grow on other crystallographic faces, starting with a (001) substrate. In this communication, we examine the strain tensor and band-edge variation as the substrate orientation is changed, keeping the misfit constant. We will examine both a typical direct band-gap film $\left(\mathrm{In}_{0.2} \mathrm{Ga}_{0.8} \mathrm{As} /\right.$ $\mathrm{GaAs})$ and an indirect band-gap film $\left(\mathrm{Si}_{0.8} \mathrm{Ge}_{0.2} / \mathrm{Si}\right)$. For the purpose of reference throughout this work, the relevant material parameters used are given in Table I. The material parameter values in this table are obtained from Refs. 1 $\left(\mathrm{Si}_{0.8} \mathrm{Ge}_{0.2}\right)$ and 2 .

The first quantity examined is the strain tensor as a function of substrate orientation. This is straightforward to obtain if we note that the in-plane lattice constant of the overlayer is the same as the substrate. Details of the calculation are reported elsewhere. ${ }^{1}$ In Fig. 1 the strain tensor components are shown for different substrate orientations, and are plotted in units of lattice misfit, $\epsilon_{\|}$. These data are obtained as a solution of Hooke's law, subject to the condition that the stress causing the distortion is applied to the film only in the plane of the substrate.

As can be seen, for (001) orientations, the tensor is diagonal with

$$
\begin{aligned}
& \epsilon_{x x}=\epsilon_{y y}=\epsilon_{\|}, \\
& \epsilon_{z z}=-\epsilon_{\|} / \sigma^{(001)}, \\
& \epsilon_{x y}=\epsilon_{y z}=\epsilon_{z x}=0,
\end{aligned}
$$

where $\sigma^{(001)}$ is Poisson's ratio: $\sigma^{(001)}=\frac{1}{2} c_{11} / c_{12}$. However, off-diagonal shear components emerge as one goes to other orientations. Here $C_{11}$ and $C_{12}$ are the elastic stiffness constants.

To study the effect of the strain tensor on the various high symmetry points in the Brillouin zone, we examine the conduction-band edges for a direct gap material and an indirect gap material. The shifts in the band edges are given by the Hamiltonian for the deformation potential perturbation. The Hamiltonian of this perturbation, $H_{\epsilon}$, has matrix elements of the form

$$
H_{\epsilon, i j}=\sum_{\alpha, \beta=1}^{3} D_{i j}^{\alpha \beta} \epsilon_{\alpha \beta},
$$

where $\epsilon_{\alpha \beta}$ is the $(\alpha, \beta)$ element of the strain tensor and $D_{i j}^{\alpha \beta}$ is a deformation potential. Since $D_{i j}^{\alpha \beta}$ bears the subscripts $(i, j)$, it may be thought of as a matrix element of an operator $D^{\alpha \beta}$, and it is the same way that $H_{\epsilon, i j}$ is a matrix element of the operator $H_{\epsilon}$. Since there are nine strain tensor elements $\epsilon_{\alpha \beta}$, there are correspondingly nine deformation potential operators $D^{\alpha \beta}$. However, as $\epsilon_{\alpha \beta}$ is symmetric, $D^{\alpha \beta}$ is also symmetric $\left(D^{\alpha \beta}=D^{\beta \alpha}\right)$, leaving a maximum of only six independent operators.

For the direct band-gap conduction band the shift in the position of the $(000)$ edge is

$$
\delta E^{(000)}=\Xi_{d}^{(000)}\left(\epsilon_{x x}+\epsilon_{y y}+\epsilon_{z z}\right) .
$$

For the (100)-type indirect material, the shifts are

$$
\begin{aligned}
& \delta E^{(100)}=\Xi_{d}^{(100)}\left(\epsilon_{x x}+\epsilon_{y y}+\epsilon_{z z}\right)+\Xi_{u}^{(100)} \epsilon_{x x} \\
& \delta E^{(010)}=\Xi_{d}^{(100)}\left(\epsilon_{x x}+\epsilon_{y y}+\epsilon_{z z}\right)+\Xi_{u}^{(100)} \epsilon_{y y} \\
& \delta E^{(001)}=\Xi_{d}^{(100)}\left(\epsilon_{x x}+\epsilon_{y y}+\epsilon_{z z}\right)+\Xi_{u}^{(100)} \epsilon_{z z}
\end{aligned}
$$


TABLE I. Material parameters for $\mathrm{Si}_{0.8} \mathrm{Ge}_{0.2}$ and $\mathrm{Ga}_{0.8} \mathrm{In}_{0.2}$ As.

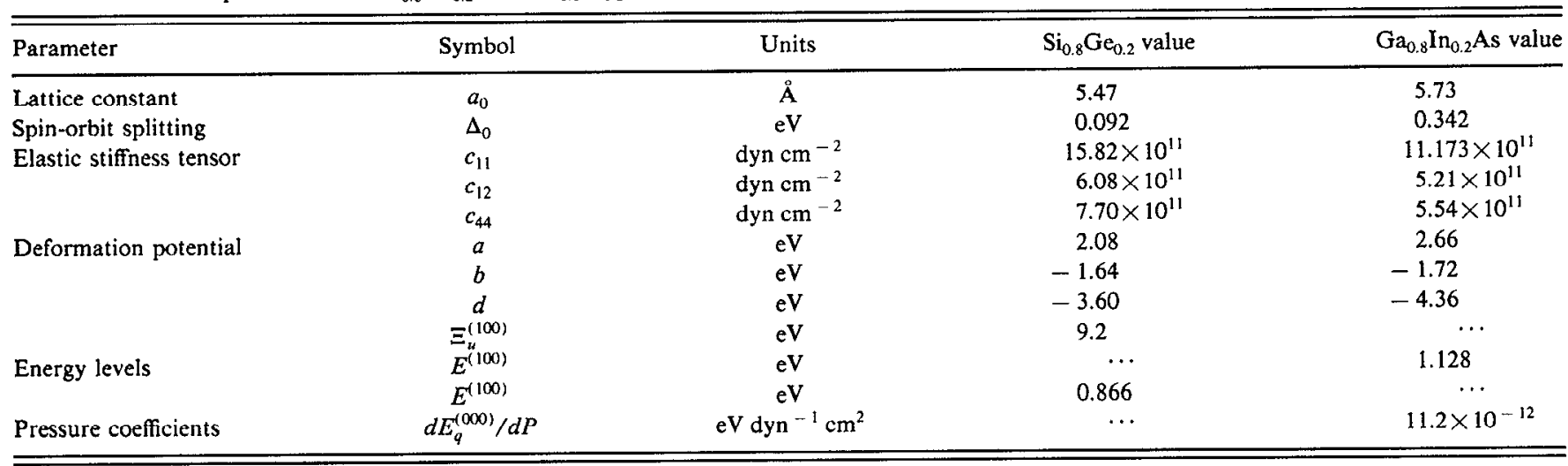

The symbols $\Xi_{d}$ and $\Xi_{u}$ represent the dilation and uniaxial deformation potentials for the corresponding valleys.

The effect of the strain on the valence-band edges is given by the shifts in the eigenvalues of the Hamiltonian $H_{\epsilon}+H_{\mathrm{so}}{ }^{1}$ where $H_{\epsilon}$ is the deformation potential perturbation Hamiltonian:

$$
H_{\epsilon}=\left[\begin{array}{cc}
H^{\prime \prime} & 0_{3 \times 3} \\
0_{3 \times 3} & H^{\prime \prime}
\end{array}\right]_{\downarrow}^{\uparrow}, \quad H^{\prime \prime}=\left[\begin{array}{lll}
l \epsilon_{x x}+m\left(\epsilon_{y y}+\epsilon_{z z}\right) & n \epsilon_{x y} & n \epsilon_{z x} \\
n \epsilon_{x y} & l \epsilon_{y y}+m\left(\epsilon_{z z}+\epsilon_{x x}\right) & n \epsilon_{y z} \\
n \epsilon_{z x} & n \epsilon_{y z} & l \epsilon_{z z}+m\left(\epsilon_{x x}+\epsilon_{y y}\right)
\end{array}\right]^{x} \begin{aligned}
& x \\
& y
\end{aligned}
$$

and $H_{\text {so }}$ is the spin-orbit coupling perturbation Hamiltonian:

$$
H_{\mathrm{so}}=\frac{\Delta 0}{3}\left[\begin{array}{cccccc}
0 & -i & 0 & 0 & 0 & 1 \\
i & 0 & 0 & 0 & 0 & -i \\
0 & 0 & 0 & -1 & i & 0 \\
0 & 0 & -1 & 0 & i & 0 \\
0 & 0 & -i & -i & 0 & 0 \\
1 & i & 0 & 0 & 0 & 0
\end{array}\right] \begin{aligned}
& x \uparrow \\
& y \uparrow \\
& z \uparrow \\
& x \downarrow \\
& y \downarrow \\
& z \downarrow
\end{aligned} .
$$

In Eqs. (5) and (6), $l, m$, and $n$ are the valence-band deformation potentials while $\Delta_{0}$ is the spin-orbit splitting.

In Fig. 2 we examine the variation with substrate orientation of the band gap of $\mathrm{In}_{0.2} \mathrm{Ga}_{0.8}$ As grown pseudomorphically on GaAs. The overlayer has a $1.4 \%$ misfit with the substrate. The band gap of the relaxed overlayer is $1.128 \mathrm{eV}$ at $300 \mathrm{~K}$, and is shown as a dashed line for comparison. The band gap increases to $1.218 \mathrm{eV}$ when the layer is grown pseudomorphically on $\mathrm{GaAs}(001)$. From this point, the gap increases, to its maximal value of 1.24 $\mathrm{eV}$, as the orientation is changed toward the (111) orientation. As the orientation is varied from the (111) direction to the (101) direction, the band gap decreases slightly by $10 \mathrm{meV}$. If the pseudomorphic layer is grown as a quantum well, one has to include the confinement effects which will add an additional offset to the band gap.

In Fig. 3 we show the result of similar calculations for $\mathrm{Si}_{0.8} \mathrm{Ge}_{0.2}$ grown on a $\mathrm{Si}$ substrate. Again, the value of the indirect band gap of the unstrained alloy is shown as a dashed line for comparison. In this case, since the sixfold $\Delta$-type conduction-band edge splits due to the strain, we show the results for the smallest band gap as well as the gap due to the upper [001]-type valley.
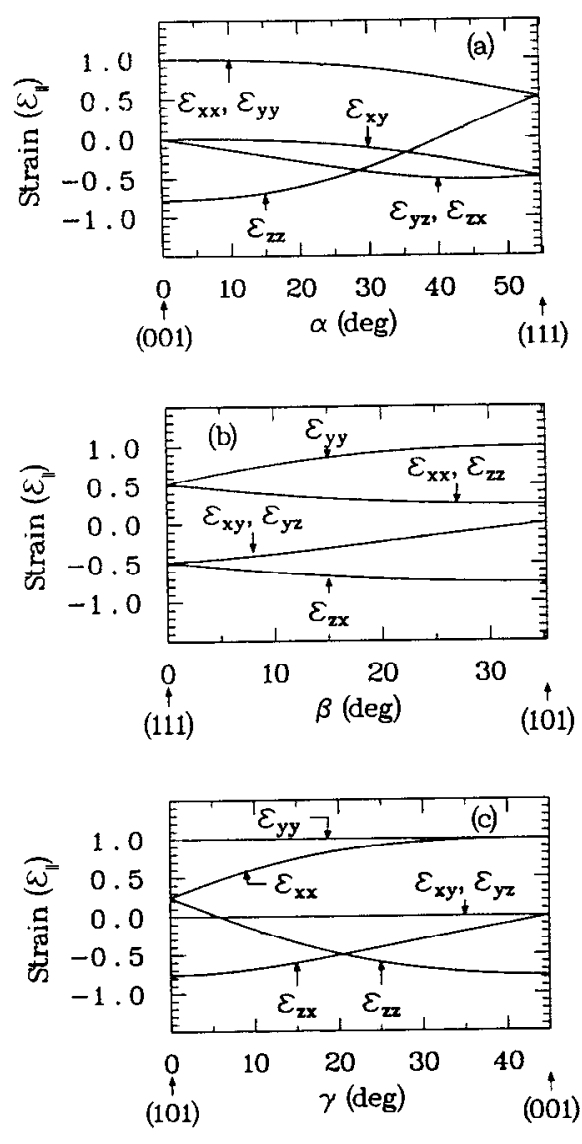

FIG. 1. Strain tensor component variation with substrate orientation. Components are plotted in units of $\epsilon_{\|}$. (a) From (001) to (111); the angle between [001] and [111] is $\alpha_{\max }=54.736^{\circ}$. (b) From (111) to (101); the angle between [111] and [101] is $\beta_{\max }=35.264^{\circ}$. (c) From (101) to (001); the angle between [101] and [001] is $\gamma_{\max }=45^{\circ}$. 

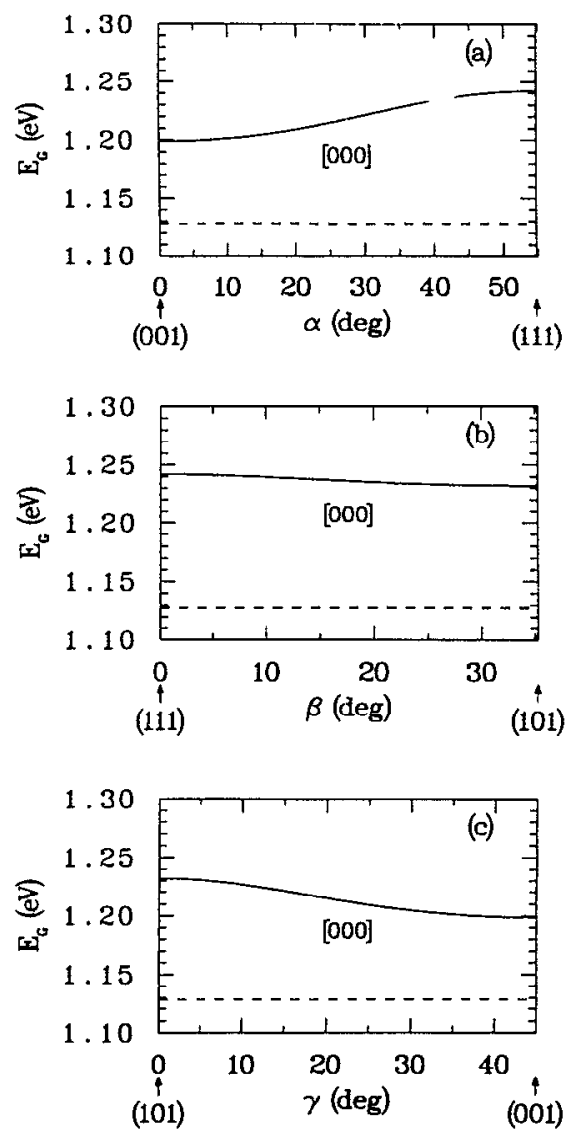

FIG. 2. Variation with substrate orientation of direct band gap of $\mathrm{In}_{0.2} \mathrm{Ga}_{0,8} \mathrm{As} / \mathrm{GaAs}$ (solid lines). Band gap in unstrained $\mathrm{In}_{0.3} \mathrm{Ga}_{0.8} \mathrm{As}$ (dashed line). (a), (b), (c) Orientations as in Fig. 1.

In keeping with the negative hydrostatic pressure coefficient of $\Delta_{1}$-type levels, the band gap in $\mathrm{Si}_{0.8} \mathrm{Ge}_{0.2}$ is found to decrease under in-plane contraction. This is contrary to the behavior of the direct band gap in $\mathrm{In}_{0.2} \mathrm{Ga}_{0.8} \mathrm{As}$ under similar distortion. As in the case of $\mathrm{In}_{0.2} \mathrm{Ga}_{0.8} \mathrm{As}$, the band gap of $\mathrm{Si}_{0.8} \mathrm{Ge}_{0.2} / \mathrm{Si}$ is greatest for a (111) substrate and least for a (001) substrate. The band gap for a (101) substrate is very slightly greater than for a (001) substrate. Depending upon the substrate orientation, the splitting between the $\Delta_{1}$ valleys may be larger [e.g., (001) substrate] or smaller [e.g., (111) and (101) substrates] than the strain-induced band-gap shift.

Strain-induced changes to a direct band gap, such as that of $\mathrm{In}_{0.2} \mathrm{Ga}_{0.8} \mathrm{As}$, will primarily affect the optical properties of the material, leaving the electron transport characteristics virtually unchanged (for low electric fields). However, the splitting in addition to the shift of the indirect conduction-band edge in $\mathrm{Si}_{0.8} \mathrm{Ge}_{0.2}$ will result in an effect to both the optical as well as the electronic transport characteristics. Under suitable substrate orientations, the conduction-band-edge effective density of states will be reduced by a factor of nearly $\frac{1}{3}$ or $\frac{2}{3}$. The relative raising of selected $\Delta_{1}$ valleys under strain may introduce an anisotropy to the transport characteristics. ${ }^{3}$ While this effect is greatest for the (001) substrate, other substrate orienta-
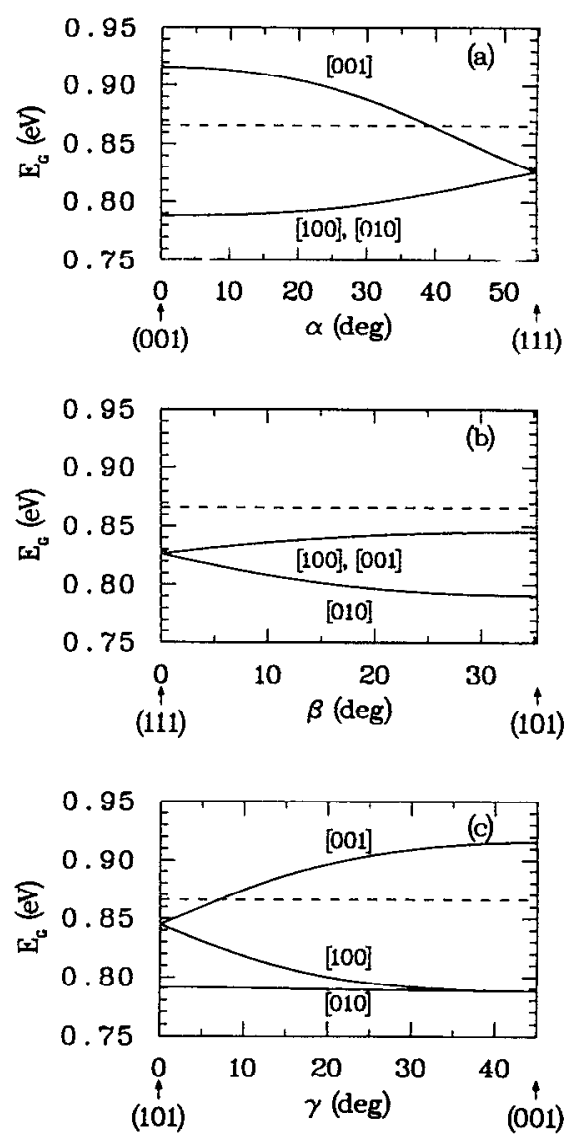

FIG. 3. Variation with substrate orientation of indirect band gaps of $\mathrm{Si}_{0.8} \mathrm{Ge}_{0.2} / \mathrm{Si}$ (solid lines). Band gap in unstrained $\mathrm{Si}_{0.8} \mathrm{Ge}_{0.2}$ (dashed line). (a), (b), (c) Orientations as in Fig. 1.

tions may be employed to either increase or decrease the electron conductivity effective mass for a given device structure. For example, with a (001) substrate, it is well known that the perpendicular conductivity effective mass decreases while the in-plane conductivity effective mass increases. From Fig. 3 it is seen that the conductivity effective mass for a film on a (111) will remain unchanged in all directions while the band gap decreases. For a (101) substrate, the conductivity effective mass decreases for perpen-

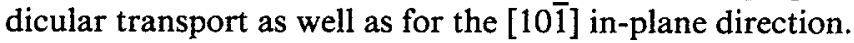
In the [010] in-plane direction, the conductivity effective mass increases.

In summary, we have examined the band gap and conduction-band degeneracy variation as a function of substrate orientation. Since the misfit is maintained constant, by properly patterning a starting substrate, tunable variation in band gap and material properties can be obtained.

This work was supported by the U.S. Army Research Office (Grant No. DAAL03-87-K-0007) and a grant from the IBM Corporation.

'J. M. Hinckley and J. Singh, Phys. Rev. B 42, 3546 (1990).

${ }^{2}$ M. Neuberger, Handbook of Electronic Materials (Plcnum, New York, 1971).

${ }^{3}$ J. M. Hinckley, V. Sankaran, and J. Singh, Proceedings IEEE/Cornell Conference on Advanced Concepts in High Speed Semiconductor Devices and Circuits, 141 (1989). 